\title{
En la era de la desigualdad, los jóvenes como protagonistas. Construyendo puentes de integración entre la educación. Las perspectivas de empleo y los procesos de participación en Argentina ${ }^{1}$
}

\author{
Pablo Christian Aparicioº \\ Sergio Ignacio Carbajal ${ }^{3}$
}

\begin{abstract}
Resumen
En el ámbito de las políticas públicas en Argentina, los jóvenes aparecen cada vez más definidos como actores estratégicos del desarrollo y en su figura se deposita la sostenibilidad y la realización del proyecto histórico y social.

Sin embargo son los mismos jóvenes quienes experimentan serias dificultades al momento de gestionar sus proyectos biográficos e itinerarios educativos y laborales.

En un contexto dominado por la expansión de las desigualdades sociales, las carencias de expectativas futuras, la invisibilización política y la postergación institucional. En este mismo escenario la actual política
\end{abstract}

1 El presente artículo se fundamenta en los hallazgos científicos y las conclusiones preliminares extraídas del Proyecto de Investigación N 1410 (Consejo de Investigación de la Universidad Nacional de Salta-Argentina) denominado "La construcción de la cultura institucional educativa en contexto social crítico. El entorno de empobrecimiento y sus consecuencias en la dinámica institucional. Estudios de caso en la ciudad de Salta" que se encuentra en las últimas instancias de ejecución, cuya dirección está a cargo del Lic. Sergio Ignacio Carbajal.

2 Dr. en Ciencias de la Educación, Profesor interino en el Departamento de Pedagogía Social del Instituto de Ciencias de la Educación de la Eberhard-Karls Universität Tübingen (Alemania), Miembro del Centro de Investigaciones Socio-Educativas del Norte Argentino (Salta/Argentina) y del Consejo de Investigación de la Universidad Nacional de Salta (Argentina) e Investigador Post-doctoral en la Unidad de investigación del Instituto de Ciencias de la Educación de la Universidad de Salamanca (España).

3 Licenciado en Ciencias de la Educación: Pontificia Universidad Javeriana. Facultad de Educación. Bogotá, Colombia, Docente en la Escuela de Ciencias de la Educación y Docente del Profesorado en Ciencias Económicas y en Ciencias Jurídicas, para graduados. Facultad de Ciencias Económicas, Jurídicas y Sociales de la Universidad Nacional de Salta. Director del Proyecto de Investigación. N 1410 Consejo de Investigación UNSa. Director de la Carrera de Especialización de Posgrado en Análisis Institucional. Res. CS. 303/03 Sede Regional Tartagal. Secretario de Extensión Universitaria de la Universidad Nacional de Salta. 
Construyendo puentes de integración entre la educación. Las perspectivas de empleo y los procesos de participación en Argentina - Pablo Christian Aparicio y Sergio Ignacio Carbajal

educativa se muestra insuficiente para afrontar los nuevos desafíos inherentes a la participación, la cohesión y la inclusión social plena.

Por este motivo, en el presente artículo se reflexionara sobre la Educación en términos de una herramienta de transformación social capaz de dinamizar procesos de inclusión social y empoderar los intereses y las demandas de los jóvenes.

Palabras clave: Desigualdad socioeducativa, Jóvenes, Instituciones educativas, Participación, Reforma educativa en Argentina.

\begin{abstract}
In the area of public policy in Argentina, young people are increasingly defined as strategic actors of development and its sustainability set is deposited and the realization of social and historical project.

But it is the same young people who experience serious difficulties when managing their biographical projects and education and employment pathways.

In a context dominated by the expansion of social inequalities, lack of future expectations, the political invisibility and institutional delay. In this scenario the current educational policy to be inadequate to meet the new challenges inherent in participation, social cohesion and inclusion full. Therefore, in this article reflect on education in terms of a tool capable of energizing social transformation processes of social inclusion and empower the interests and demands of young people.
\end{abstract}

Key words: Inequality rehabilitative, Youth, Educational Institutions, Participation, Educational Reform in Argentina. 


\section{Introducción}

La concomitante relación sostenida entre el trabajo, la educación y los jóvenes se inscribe como uno de los temas más preocupantes dentro del actual debate político social no sólo en Argentina, sino en todos los países de la región y del mundo.

Indudablemente la búsqueda de puntos de interconexión entre el mundo del trabajo, las ofertas de formación educativa y los horizontes de integración social de las nuevas generaciones representa un aspecto neurálgico del actual modelo histórico de desarrollo.

Diversos estudios educativos, laborales y económicos realizados sobre los problemas de integración de los jóvenes en Argentina evidencian que las causas y las consecuencias de la ruptura entre el trabajo, la educación y los jóvenes, develan por un lado, la limitación de la efectividad de las políticas educativas vigentes entendidas como promotoras de ascenso social y bienestar económico. En este sentido, las credenciales educativas adquiridas (disposiciones, conocimientos y capacidades cognitivas, profesionales y técnicas) no garantizan a priori y per se la superación exitosa de los retos vinculados a la integración social y laboral en un mercado de trabajo fuertemente restrictivo, versátil e informal.

Por otro lado, la disociación revela también la laxitud y la precariedad de las fuerzas regulatorias del Mercado, especialmente de su poder coptativo y regenerativo inherente a los procesos de inclusión en el sistema de trabajo y la participación en el mercado de consumo (Bourdieu, 1999; Bauman, 2003). Como se observara, este problema se exacerba ante la volatilización de la figura del Estado como ente regulador y veedor de la cohesión social y la ausencia de medios alternativos de inclusión social, política e institucional.

En la actualidad y ante la impronta avasallante de fenómenos sociales como el desempleo y el subempleo; la pobreza y las 
nuevas dinámicas de empobrecimiento; la consolidación de los procesos de segregación étnica-cultural y los contextos de vulnerabilidad social; la degradación de las condiciones de trabajo; y la restricción del acceso a los bienes materiales y simbólicos requeridos para la participación social plena, no se evidencian en el país -en general- y en la provincia de Salta -en particular- la existencia de propuestas integrales que hayan aportado a la resolución de estas restricciones.

Por este motivo en el presente artículo se reflexionará sobre la pertinencia de la oferta educativa en razón a las demandas de los jóvenes al momento de organizar sus trayectorias educativas y laborales, especialmente de aquellos sujetos más expuestos a la exclusión social, la pobreza y la falta de reconocimiento político e institucional.

\section{El carácter estructural de las transformaciones sociales en los 90 y su impacto en los albores del siglo XXI}

A través de la implementación de múltiples reformas políticas en el terreno educativo, social, laboral, jurídico, tecnológico y científico concretadas en el marco de la reestructuración del Estado se pusieron en medidas tendientes a la privatización de los servicios públicos y la supresión provisoria y definitiva de estructuras y funciones públicas en materia de gestión, financiamiento y regulación de políticas sociales. Estas tres ideas centrales fueron en muchos casos establecidas como precondiciones ineludibles para la instauración de la disciplina fiscal, la eficiencia administrativa, la idoneidad en el manejo de los recursos fiscales y la consagración de los valores de la equidad y la eficiencia.

Ahora bien, desde estos axiomas se abogó por la reducción y el saneamiento del déficit público y se procedió a reestructurar la mayoría de las instituciones y las dependencias públicas encargadas de brindar servicios en el área de salud, seguridad social, educación, comunicación, transporte, etc. 
La reducción del gasto público, la desafiliación y desautorización del Estado como principal regulador y garante del bienestar social y la paralela -y abrupta- institucionalización del Mercado como nuevo axioma de ordenamiento social constituyen en este escenario las transformaciones más significativas sobre las cuales se edificó la sociedad argentina de los 90 y de los albores del nuevo siglo (Oszlak, 2003).

Actualmente el escenario socio-histórico está fuertemente “determinado por múltiples contradicciones, inviabilidades y progresivas dinámicas de exclusión, suscitadas por la proyección de los tópicos políticos neoliberales, cuyos resultados han incrementado y legitimado la segregación y la polarización social, fundada en la distribución desigual de los bienes materiales, culturales y sociales. Y esto se debe principalmente a que las modificaciones políticas fueron implementadas en un corto período temporal y con una lógica de carácter coercitivo y unidireccional que no contempló como necesario propiciar procesos de adecuación destinados a la localización in situ de las reformas en los diversos contextos sociales, para poder identificar y aprehender con mayor fidelidad tanto a) la dimensión real de las demandas y necesidades de los sectores sociales e individuos como b) las condiciones materiales, tecnológicas, políticas e institucionales de las jurisdicciones federales (provincias, municipios) y la complejidad del espacio social, la pluralización de las condiciones de vida a nivel familiar, social, ocupacional, demográfica, etc.), a donde el conjunto de los postulados de la reforma fueron introducidos."

La brecha social planteada -casi antagónicamente- entre sectores ricos y pobres, con una clase media en paulatino desgranamiento, ha tendido a incrementarse sin poder encontrar en la actualidad una malla de contención o políticas tendientes a la compensación de los déficits sociales acumulados, vale decir, dotadas de la capacidad de contrarrestar la incertidumbre, la in-

4 Aparicio, 2007b. Op. cit. pág. 44. 
Construyendo puentes de integración entre la educación. Las perspectivas de empleo y los procesos de participación en Argentina - Pablo Christian Aparicio y Sergio Ignacio Carbajal

seguridad, el desmedro de las redes tradicionales de integración cívica y la "centrifugación social". ${ }^{5}$

Con la introducción de reformas y programas políticos de naturaleza estrictamente económica el sistema educativo argentino -entendido como cuerpo e instancia de organización social de los bienes de la cultura- sufrió una fuerte desestabilización y crisis que estuvo conjugada al debilitamiento del Estado y la limitación de su intervención directa en la distribución de recursos financieros y técnicos, la administración burocrática y la regulación de los servicios sociales.

Desde las nuevas disposiciones políticas encarnadas en la Ley Federal de Educación (24.195) se propició la desjerarquización paulatina de las acciones, los programas y los dispositivos circunscriptos en el área pública, puesto que desde el nuevo programa político la intromisión de los actores privados en la administración del servicio educativo y la regencia de las lógicas de competitividad y eficiencia se concebían como los motores genuinos de la transformación educativa y social en curso.

En esta perspectiva también, la educación encarnaba un factor determinante del desarrollo socioeconómico siempre y cuando acompañase de forma supeditada y congruente la consecución de los objetivos trazados por las políticas y las demandas económicas, financieras y laborales imperantes, vale decir sin alterar el curso armónico y autorregulable de dichos procesos.

La instauración de nuevos criterios de gestión, administración y evaluación de los servicios sociales y educativos se basaban en las máximas de rentabilidad, la equiparación y regulación

5 "La centrifugación social (como desarticulación del modelo incluyente) (Villareal, 1996), la privación de lo político y la preeminencia de la economía divorciada de la ética del bien común constituye rasgos decisivos del escenario argentino de los 90. La heterogeneidad y la diversidad de la exclusión se expresa en su ataque cruzado a lo largo de toda la estructura social: no todos quedan simultáneamente afuera, ni con la misma severidad. Las combinaciones de exclusión son variadas." En Mancebo (1999), Op. cit. pág. 183. 
entre el costo y el beneficio económico, el retorno positivo de la inversión, la descentralización de las administraciones públicas, la apuesta por una mayor autonomización de las decisiones y la atribución individualizada de las responsabilidades en torno al rendimiento escolar y académico de las personas fueron algunos de los pilares fundamentales de la nueva propuesta educativa inspirada en el modelo de pensamiento neoliberal.

En consideración al neoliberalismo, Bourdieu (1998) arguye que "este movimiento (de transformación mundial), posibilitado por la política de las desregulaciones financieras, orientado hacia la utopía neoliberal de un mercado puro y perfecto, se implementa mediante la acción transformadora y, es menester decirlo, destructora de todos los recursos políticos (...) buscando cuestionar toda estructura colectiva capaz de ser un obstáculo a la lógica del mercado puro. El programa neoliberal (...) tiende globalmente a favorecer la censura entre la economía y las realidades sociales y a construir así en la realidad un sistema económico en conformidad a la descripción teórica, es decir, una suerte de máquina lógica que se presenta como una cadena de situaciones forzosas que arrastran a los agentes económicos". ${ }^{6}$

La disociación reduccionista y antagónica planteada entre las prioridades del discurso económico y el resto de los discursos sociales (éticos, culturales y políticos) torna a la realidad en un fenómeno harto complejo, paradójico y taxativo, desde donde se reproducen las rupturas y las contradicciones sociales (Bourdieu, 1998b). La exaltación de la "lógica del sistema" se condice con el carácter regresivo y ahistórico de los planteamientos neoliberales, que en un contexto en donde prevalecen la desigualdad social, la precariedad y la inflexión generalizada de las bases democráticas de la vida institucional, sólo propone la lógica del mercado como alternativa unívoca para la superación de las dificultades estructurales que atisban a la sociedad (Beck, 1990).

6 Op. cit. pág. 3. 
Por esta razón, algunos investigadores como Riquelme (2004), Filmus et al. (2001) y Zaffaroni y Carbajal (2008) sostienen que la unidireccionalidad y el reduccionismo del modo en que desde los planteamientos neoliberales se concibe el desarrollo económico y social, deviene en utópico, puesto que los beneficios y las ventajas que provee el crecimiento económico y la solidez financiera en una sociedad cada vez más polarizada al no sopesar ni doblegar la expansión de la desigualdad y la segregación social acaba reproduciendo las lógicas de un sistema social excluyente y meritocrático.

En esta perspectiva, la reducción y la negación de formas de organización social alternativas al modelo hegemónico refuerzan la idea de la insuperabilidad de la lógica de mercado pretendida como universalmente válida y extrapolable, que devino también en la exaltación del pensamiento único y reificante, que sesgó -tergiversando y diluyendo- la gestación de formas de entendimiento crítico frente a la realidad histórica y social (Bauman, 1999). Así pues, este pensamiento reificante y subyacente en los postulados ideológicos neoconservadores subsume el valor ontológico de la alteridad y la diversidad cultural provocando la cosificación y la banalización de la diversidad, lo heterogéneo y lo distinto en términos de posibilidad, viraje o divergencia.

La incorporación de postulados economicistas y pragmáticos también en el campo de la gestión de las políticas educativas y sociales, propició la puesta en práctica de reduccionismos y disociaciones que obstaculizaron el reconocimiento de la complejidad y la heterogeneidad inherente a la realidad educativa. Este hecho quedó plasmado en los objetivos y los estilos de organización, planificación e inversión pública prevaleciente en los últimos 10 años.

Al presente, las luchas de los movimientos sociales y algunas decisiones del gobierno nacional, han logrado producir algunas modificaciones, pero sin alterar de fondo la estructura de la reforma neoliberal implementada. Tal vez, en otros aspectos, se deba a 
la permanencia de muchos de los ejecutores del proyecto neoliberal en cargos estratégicos y nodales dentro de la administración nacional y del Parlamento a nivel nacional y provincial.

\section{La política educativa entre el ímpetu de la reforma y el ocaso de la transformación esperada}

La reforma educativa de los '90 propició la introducción coercitiva y discontinua de métodos, teorías, tecnologías, programas de formación, arreglos institucionales y propuestas curriculares -autoproclamadas como "innovadoras y eficientes"- que en los hechos, dado su carácter coercitivo y exógeno, desdeñaron la posibilidad de diálogo y de concertación colectiva con el conjunto de actores e instituciones que conforman la comunidad educativa al momento de definir los puntos y los objetivos más relevantes de la nueva agenda política.

Dada la consagración de la economía de mercado y la instauración de "Estado minimizado" desde el espacio educativo se han intentado generar mecanismos de socialización buscando amalgamar las tendencias y las demandas correspondientes al ámbito económico (mercado, empresas, industrias, servicios, etc.) y al ámbito político educativo (escuelas, institutos de formación e instrucción técnica, profesional, programas y proyectos de capacitación, universidades, etc.).

Al día de hoy, este proceso se sigue efectivizando en Argentina y América Latina -salvo escasas excepciones- sin obtener resultados alentadores en términos de una transformación "de base" y no sólo "de forma" que avancen en la superación de la exclusión, la marginalidad y la no-participación planteadas dentro y entre las diferentes estructuras e instituciones correspondientes al mundo educativo, laboral y económico (Aparicio, 2008).

En especial consideración a la participación restringida y volátil de los sectores juveniles mas segregados y desaventajados del sistema educativo -población objetivo en nuestro proyecto de 
investigación- se destaca la necesidad de repensar la importancia de la educación en tanto espacio de reflexión política y lucha de intereses, y bisagra de articulación institucional y social potenciadora de las transformaciones culturales, científicas, técnicas, éticas y estéticas requeridas.

En este sentido, la superposición y la disociación entre los propósitos políticos del campo educativo en relación a las propuestas y las ofertas del campo económico laboral acentúa las desigualdades planteadas en las posibilidades objetivas de participación que divide a los grupos sociales beneficiados y mejor posicionados pertenecientes a la clase media alta y alta, de aquellos grupos más desfavorecidos -excluidos que integran la clase media baja, baja en regresión y baja-pobre.?

En este contexto se torna imperioso reestructurar los parámetros de evaluación, diseño y planificación didáctica y curricular para corregir las insuficiencias y las debilidades inherentes a las actuales ofertas educativas y a los criterios pedagógicos con el propósito de abordar el tratamiento de problemas complejos como la desigualdad económica, cultural y social que a posteriori en el caso de los jóvenes se proyectan en el mercado laboral (Naciones Unidas, 2005; CEPAL y CELADE, 2000).

En virtud de la pertinencia de los medios de socialización y formación educativa se abren diferentes interrogantes referidos primordialmente a la concepción adecuada de los procesos educativos para poder influir favorablemente en la inclusión social y laboral de los jóvenes y que están referidos a las siguientes cuestiones:

7 A modo de contrastación crítica sobre el valor trascendente de la participación, Frigerio (2002) postula que "ser par y tener parte, ser par y formar parte son modos de entender una ciudadanía plena. (...) El economicismo neoliberal y las políticas que lo representan no consideran sinónimos ser y tener parte. Los sin parte, los que no cuentan con pasaporte alguno para transitar libremente por el territorio de la dignidad, resultan funcionales a las políticas que los asignan en el lugar de los supernumerarios, sobrantes, excedentes, aquellos para quienes no se reconoce ningún gesto de la humanidad". Op. cit. pág. 57. 
¿Cómo se debería educar adecuadamente a los jóvenes para poder apoyar la realización de sus trayectorias de vida a nivel biográfico y social?; ¿cuáles deberían ser los métodos de enseñanza y aprendizaje, las mediaciones institucionales y las propuestas curriculares y didácticas, que sirvan para la concretización de un servicio de calidad eficiente y en apertura constante a las características de los grupos y los individuos?; ¿cómo generar una instancia de diálogo y cooperación en el área de formación de jóvenes entre el conjunto de acciones y programas destinados desde diferentes carteras públicas y que persiguen análogamente el mejoramiento de la calidad de vida de este sector?

El distanciamiento crítico planteado entre el sistema educativo (instituciones de formación), los múltiples escenarios de vida de los jóvenes y el sistema de empleo (mercado de trabajo), reclaman del Estado la elaboración de una estrategia amplia y cohesionante, que permita superar la disposición antagónica y paralela prevaleciente entre los diferentes campos políticos sociales y sus respectivos intereses, condiciones y perspectivas de acción.

\section{El escenario educativo: la vigencia de las desigualdades, las deudas por saldar}

La actual política educativa argentina preconiza a nivel teórico y conceptual por un mayor compromiso social que el sistema educativo y el conjunto de los dispositivos institucionales tienen que asumir previamente para posibilitar la transformación de las condiciones sociales de desigualdad y exclusión regentes. Sin embargo, en la práctica concreta parecen no existir pruebas contundentes que demuestren que se esté trabajando en la erradicación de las dinámicas, los procesos y las estructuras políticas excluyentes y segregatorias que tienden a reproducir un orden social desigual.

En este contexto se instaura una de las grandes paradojas de la Argentina de hoy en la que se combina la democracia política con la exclusión social, cultural y económica progresiva. 
Apoyándonos en autores como Tenti Fanfani (2003), Tiramonti y Minteguiaga (2004), Tedesco (2004) y Salvia (2002), se podría sostener que en la implementación de la reforma política educativa en Argentina al igual que en muchos otros países de la región prevaleció ante todo una lógica burocrática-financiera inspirada en una racionalidad instrumental y economicista.

Desde la lógica burocrática-financiera avalada por los organismos de cooperación y financiamiento internacional se privilegiaron los objetivos vinculados a la reestructuración presupuestaria de la educación, la desarticulación de las administraciones y las burocracias implicadas en los procesos de decisión, gestión y planificación; y finalmente la privatización del servicio público en donde el mercado aparecía como un administrador eficiente, neutral y equitativo, capaz de subsanar el funcionamiento deficitario del Estado en materia de administración y gestión de las políticas educativas. Al respecto Filmus y Miranda (1999:144) postulan que "las restricciones a la inversión educativa, las difíciles condiciones de trabajo de los docentes, las lógicas del ajuste que predominan en un conjunto de cambio, la carencia de condiciones sociales de educabilidad mínimas de una gran cantidad de alumnos, y la dificultad para avanzar en procesos de concertación educativa son algunos de los condicionantes más importantes que el contexto impone a las transformaciones educativas."

Las recomendaciones y directrices políticas y técnicas emitidas por diferentes organismos internacionales de financiación y desarrollo condicionaron, y en algunos casos, determinaron la transpolación de los nuevos axiomas de gestión, administración, evaluación y organización de la política educativa en diferentes países de la región. Sobre estas directrices se buscó establecer una sintonía armónica entre los proyectos educativos locales y los premisas políticos innovadoras -erigidas desde los centros de planificación y administración financiera- que abogaban por el fortalecimiento de los procesos de descentralización, la incorporación de mecanismos mensurables y estándares de evaluación 
de la calidad educativa, la renovación sistemática de los contenidos curriculares, la instauración de un modelo focal-coyuntural de planificación y desarrollo curricular y la responsabilización creciente de la comunidad en torno al sostenimiento económico de la oferta escolar y la pertinencia social del proyecto educativo en razón a las demandas y las características locales.

A pesar de tratarse de objetivos amplios y con cierto tinte progresista, estas prerrogativas desafortunadamente contemplaron de modo incompleto la diversidad y la singularidad de los contextos culturales y materiales inherentes a la educación así como tampoco sus habitus y estilos organizacionales de los actores sociales y que condicionan el trabajo educativo y su respectiva impronta social.

La priorización de la lógica burocrático-financiera trajo como consecuencia el descuido y la desatención de problemas y asuntos de carácter estrictamente "educativo" como por ejemplo las debilidades y las anomias registradas en el ámbito curricular, la formación profesional adecuada, la organización institucional, la capacitación docente, la investigación educativa, los procesos de evaluación, la planificación estratégica, la contextualización curricular, la toma de decisión local y el empoderamiento de las instituciones y las comunidades educativas, etc. (Álvarez y Aparicio, 2000).

Así pues, la hegemonía de la lógica burocrático-financiera se produjo en menoscabo de una lógica política-pedagógica considera como premisas de todo proceso de reforma el refuerzo concerniente a la calidad de la oferta, la diversificación de las estrategias de compensación socio-educativa y la ampliación del contenido democrático y participativo en la elaboración, discusión e implementación de las propuestas y los programas de formación.

Por su parte, Puiggrós y Dussel (1999: 16-17) añaden que “el modelo neoliberal (...) produce una crisis orgánica del sistema educativo. Fractura los vínculos constitutivos de la organización 
político-institucional, de las diferencias generacionales y sociales y de la cadena de transmisión de la cultura. (...) La escuela está cada vez más atravesada por otras dinámicas culturales, que la están desplazando en importancia y en productividad (...). Las políticas neoliberales redefinen los espacios públicos $\mathrm{y}$, entre ellos, el escolar es uno de los más afectados. La escuela es más pobre, más chica, menos importante."

La sobreestimación taxativa de lo económico en menoscabo de lo educativo y lo social, ha obstaculizado la posibilidad de engendrar criterios democráticos y participativos, capaces de refrendar y superar la exclusión social fuertemente constreñida en los mismos procesos de socialización y formación escolar.

Por todo, la actual crisis educativa expone la necesidad de buscar nuevas orientaciones políticas, filosóficas y epistemológicas que den sentido a la acción educativa y sirvan de referentes para la elaboración de soluciones idóneas a las múltiples limitaciones registradas en los diferentes niveles del sistema educativo, como por ejemplo:

A nivel estructural existen tendencias y lógicas implícitas en el terreno político, curricular y didáctico que favorecen la reproducción de la inequidad social, la invisibilizacion de la pluralidad cultural y subjetiva, y la perpetuación de la segmentación educativa;

A nivel del currículum y de los contenidos educativos se constata el déficit en la calidad y la especificidad de los saberes y las competencias que se transmiten a través de la escuela y que además guardan poca concatenación con las demandas y los contextos locales. Desde esta perspectiva, el desfasaje y la desactualización de la propuesta curricular parece estar conjugada a la estructura política prevaleciente que insiste en la homogeneidad curricular centralizada y tradicional oculta (solapada y camuflada) tras discursos progresistas que avalan la importancia del contenido democrático, flexible y contextual de la oferta educativa. Desde este 
prisma ideológico también se asiente la diversidad en términos de desigualdad, diferenciación selectiva y desagregamiento social sin incurrir en el reconocimiento objetivante de la pluralidad y la alteridad étnica y cultural;

A nivel institucional y de los actores, la introducción de nuevos perfiles, competencias y disposiciones para los profesionales de la educación no ha estado acompañada con la puesta en vigor de un programa de capacitación y actualización sistemático, continuo y contextualizado que brindara medios y espacios idóneos para dinamizar el proceso de replanteo de la profesionalidad docente y los axiomas del quehacer pedagógico en todas las instancias del sistema educativo; $y$

A nivel de la comunidad educativa en general se requiere aún definir claramente cuál es el rol que compete a cada una de las partes implicadas en la viabilización del proyecto y del proceso educativo, a saber: los alumnos, las familias, la comunidad, el Estado, las entidades privadas, las organizaciones no gubernamentales, el sector empresario y las instituciones civiles, deportivas y eclesiásticas en vistas a conglomerar los esfuerzos, extender las oportunidades educativas para todos y empoderar las intervenciones de los diferentes actores y sectores sociales en beneficio de un acceso irrestricto a una formación de calidad. En este sentido la nueva Ley de Educación, N ${ }^{\circ}$ 26.206, denominada Ley de Educación $\mathrm{Nacional}^{8}$, en tono reivindicativo subraya la importancia de adecuar la relación entre educación, mercado y los demás ámbitos sociales y fija en los capítulos I y II, principios, criterios y fines, en orden a la igualdad de posibilidades y a la inclusión social, de forma coherente, y más integrada en el conjunto del texto. Sin embargo ha creado insatisfacciones como la cuestión presupuestaria para su funcionamiento, aspecto que fuera decisivo en la propuesta educativa precedente y que contribuyera a ahondar las desigualdades socioeconómicas y la segregación educativa. ${ }^{9}$

8 Promulgada el 27 de diciembre de 2006.

9 Título I, Capítulo I, Art. $9^{\circ}$. 


\section{Los jóvenes circunscritos en un contexto de incertidumbre y postergación}

La dificultad de construir una mayor inclusión social apoyada en la ampliación de la estructura de oportunidades, se sustenta en el deterioro e inadecuación de los sistemas políticos, jurídicos e institucionales que organizan los procesos y las estructuras de formación educativa y asistencia social. Del mismo modo, el distanciamiento entre las ofertas políticas y las demandas reales, desde las observaciones y las conclusiones derivadas de nuestros trabajos de investigación, constituye uno de los dilemas más sobresalientes que esbozan el escenario histórico de los jóvenes a donde la falta de posibilidades de participación social plena atraviesa las formas de auto-percepción (identidad), las expectativas (marco subjetivo del comportamiento) y las proyecciones sociales de los itinerarios biográficos de los sujetos juveniles (Aparicio, 2007).

El cambio de las estructuras sociales bajo el prisma ideológico neoliberal representa en el caso argentino el marco de referencia primordial desde donde concebir los nuevos procesos sociales, económicos y culturales que emergen a partir de mediados de la década del 80 extendiéndose hasta inicios del siglo XXI (Lechner, 1999). El "achicamiento del Estado" como consigna orientadora de las medidas de discontinuación y clausura de las políticas públicas constituyó el axioma de la reforma estructural. Sus consecuencias -algunas irreversibles a corto y mediano plazo- no simbolizan en absoluto un resultado fallido o no deseado del programa político implementado, sino por el contrario, su repercusión y consecuencia histórica vastamente paradójica y contradictoria. ${ }^{10}$

10 Autores como Minujín (1998) y Sottoli (1999) sostienen que a partir de las políticas neoliberales quedó establecida (instituida) la transpolación del eje conductor del desarrollo y la cohesión social que se transfiere de manos del Estado hacia la voluntad, el interés y la soberanía de los individuos y las individualidades, el liderazgo de los sectores económicos de la esfera nacional e internacional y la acción de grupos políticos selectos y monopolizadores del poder y la decisión política. 
La repercusión de las transformaciones estructurales viabilizadas a través de los procesos de privatización, desregulación y descentralización -esencialmente- evidencian resultados cuestionables en especial referencia al ámbito de las políticas públicas y el campo económico y social, entre los que pueden mencionarse: el incremento de la desocupación juvenil, la agudización de la pobreza, la expansión del riesgo social, la desigualdad de oportunidades entre las regiones, provincias y municipios, la concentración de los ingresos y la riqueza, la exacerbación de mecanismos de corrupción, el afloramiento de estructuras políticas clientelistas, la inmunidad frente al abuso del poder, el debilitamiento de los fundamentos de la vida democrática, etc.

Como ya fuera expuesto, con la pérdida de eficiencia de los espacios institucionales públicos y el desdibujamiento sistemático de las responsabilidades políticas que el Estado históricamente había contraído, se potenció el "libre albedrío" de sectores y actores sociales en lo concerniente a la definición de estrategias de adaptación y reacomodamientos frente a la nuevas características y exigencias de la sociedad neoliberal, así como a la elaboración de garantías básicas para la integración y la participación social de los bienes y servicios que determinan la inclusión plena y autónoma de los ciudadanos.

En la actualidad los jóvenes a nivel estructural están siendo cada vez más interpelados y condicionados por un campo social plagado de limitaciones y rupturas estructurales a nivel político e institucional, producto de la disolución de los marcos de referencia y organización social, que aún no han podido repararse en los ámbitos de la educación, la salud, la asistencia social, el trabajo, la vivienda, etc. (PNUD, 2004; OIT, 2004).

La ausencia de una plataforma política de confluencia compartida entre las instituciones y las políticas del Estado en relación a la gestión, la planificación, la evaluación y la ejecución de las estrategias de intervención social juvenil, representa uno de 
los dilemas más preocupantes en el área de desarrollo y promoción social, educativa y laboral de la juventud en Argentina.

Al respecto la carencia de criterios organizacionales efectivos socavan proporcionalmente la dimensión "transformativa, emergente y / o creativa" de los diversos dispositivos y mecanismos de asistencia política destinada a los jóvenes en riesgo social, educativo y económico laboral (Zaffaroni, 2006).

Los jóvenes experimentan la sensación de estar situados dentro de un escenario social y educativo sobrepoblado de programas de formación, estrategias de capacitación laboral y planes de trabajo pautados a corto plazo, que al carecer de una sistematización intrínseca y una amalgama con las tendencias del mercado de trabajo, las necesidades de la comunidad local y los propios intereses individuales, coadyuva a reforzar la disfunción e impertinencia que caracteriza a muchas de las actuales ofertas educativas de capacitación existentes en el área de juventud. ${ }^{11}$

Dentro de la conducción de las políticas públicas, la hegemonía de una "anomia consentida" obstruye la creación efectiva de mayores grados de equidad, participación social y promoción social a través de la educación. Así pues, con la aceptación pasiva de las dificultades existentes y el credo en la imposibilidad de su superación, se procede a desmedrar el aporte instituyente y transformador que históricamente encarna -o debería encarnarla propuesta educativa de formación.

El hiato planteado entre los intereses de los jóvenes, sus cosmovisiones, itinerarios educativos, referentes identitarios y colectivos y la propuesta curricular educativa ha tendido en este sentido a pronunciar la segregación de los grupos juveniles, puesto que en la invisibilización de los actores y del cuadro de necesidades reales de los actores juveniles, no sólo oculta la rea-

11 Esta apreciación se fundamenta en trabajos aportados por Gallart, 2001, OIJ, 2004; Rodríguez, 2002, 2005; Aparicio, 2003, 2005, 2005b; CEPAL et al., 2008; Salvia y Tuñón, 2002; Jacinto, 2003. 
lidad a transformar sino que además tergiversa los fundamentos sobre los que se erige y reproduce un determinado orden social.

En este escenario, los jóvenes constituyen paradójicamente los destinatarios referenciales por excelencia de programas y proyectos de apoyo y promoción educativa y laboral que se han venido ejecutando con vastas limitaciones y con un impacto más que cuestionable. Por este motivo, se puede inferir que mientras prevalezca una lectura simplificadora e inadecuada son escasas las alternativas para resolver los problemas más recurrentes situados en el ámbito formativo juvenil (Carbajal, 2005).

En nuestros días, la educación representa una acción social intrincada en la vida cotidiana, que por ende está sujeta a una dinámica de cambios permanentes e inexorablemente involucrada con los desafíos históricos y sociales que la significan. Por esta razón, la educación que los jóvenes de hoy requieren para participar plenamente de la vida social rebasa el mero cumplimiento del deber y la obligación de suministrar además contenidos, conocimientos y saberes a través del sistema educativo obligatorio y formal, que si bien es insustituible no debería clausurar ni puede abarcar el resto de posibilidades de participación de los jóvenes (Aparicio, 2007c).

Con esta mirada, se deberían emprender los debates pendientes, los incógnitos abiertos y los horizontes pensables en el terreno político educativo para poder construir mecanismos reales de superación de las condiciones de exclusión, pobreza y vulnerabilidad que restringen las posibilidades de participación de los jóvenes.

Así pues, desde una propuesta educativa orientada a los jóvenes y basada en sus preconizaciones y requerimientos debería ser factible integrar intrínseca y extrínsecamente el trabajo de las asociaciones juveniles con los valores de la vida democrática y los multívocos problemas inherentes a la integración social, educativa, económica, cultural, laboral de los jóvenes, en pos de po- 
tenciar una paulatina transformación y mejoramiento de sus condiciones históricas y materiales de vida (UNESCO, 2004, 2006; PROSUR 2005, 2005; OIT, 2004b).

Por todo lo señalado se podría sostener que el mayor desafío en el ámbito de la formación de jóvenes consiste en aproximar las estructuras políticas e institucionales educativas a las demandas de estos actores tratando de reconocer, canalizar y aprehender las necesidades, las expectativas y los problemas más preponderantes desde una doble dimensión, a saber, social e individual. Esto implica ampliar el sentido y las competencias de la propuesta educativa y así también exige explorar ámbitos novedosos, dinámicas alternativas y prácticas creativas para acometer el acto formativo de enseñanza y aprendizaje que, valga la redundancia, vayan más allá de las ofertas contempladas por el sistema formal y el formato tradicional y estándar.

\section{Repensar el espacio institucional educativo: posibilidades de cambio, vías de resistencia}

Las afirmaciones que podemos hacer no tienen carácter de generalización dadas las características del modelo teórico de investigación que desarrollamos en el estudio de dos casos de instituciones educativas singulares en el noroeste de Argentina.

Hemos podido observar y analizar teóricamente que los alumnos del nivel Secundario/Polimodal, en cuanto a las profesionales reales, vinculadas con los estudios que realizan, sufren el sesgo que demarca el contexto socio-económico de procedencia. Esta no es una novedad, dado que afirmaciones similares ya fueron sostenidas por otros investigadores de diferentes nacionalidades.

Los estudiantes que provienen de sectores económicos, con ingresos muy deprimidos, como es el caso de una de las instituciones, donde el alumnado proviene en un $80 \%$ de "asentamientos", muestran tendencia al abandono de los estudios y estos es- 
tudios no suscitan compromiso con sus acciones, por lo que son comunes las "estrategias de evasión" a las que recurren.

No manifiestan tener un horizonte laboral posible, la referencia al trabajo está cargada de dudas, de inseguridades y no faltantes de pesimismo.

Pero sería muy simplista adjudicar estos comportamientos sólo a la posición socioeconómica de los alumnos puesto que convergen una pluralidad de situaciones, tales como las condiciones en las que viven, las diferentes configuraciones de las familias, la infraestructura edilicia donde estudian, las presencia o ausencias de referencias culturales que operen como estímulo, el estilo de conducción académica y el desempeño de los docentes, los prejuicios o representaciones sociales previas sobre la institución y sus alumnos, etc.

Sin embargo, los padres mayoritariamente expresan el interés de que sus hijos e hijas avancen en los estudios y alcancen un título secundario como posibilidad de que tengan una condición de vida mejor que la que ellos les pudieron dar.

Por otra parte el estudio en diversos espacios institucionales donde se reúnen los alumnos cuyas familias tienen mayores ingresos, nos permitió comprender que, si bien se tienden a utilizar similares estrategias de evasión, y los contenidos curriculares despiertan relativos compromisos, se da una tendencia mayoritaria a concluir los estudios y a tender a carreras profesionalizantes. Consideramos que aquí están presentes los contextos culturales y las representaciones sociales que soportan la autovaloración personal. Implican también otras condiciones edilicias, proyectos, personal docente y directivo.

Sostenemos también que no se pueden establecer comparaciones de una institución con otra, debido a la complejidad de las situaciones sociales y culturales en las que acontecen, así como también causa de la diversidad de los recorridos biográficos de 
los integrantes de los grupos estudiados. Tengamos presente que siempre hablamos de condicionamientos y no de determinaciones, aunque fenoménicamente pueda parecer así.

Entendemos que estas cuestiones no pueden abordarse sólo desde las instituciones educativas. En este sentido comienza a discernirse en el nivel nacional, de un modo incipiente, la importancia de las políticas de juventud e intentar proyectos. Es llamativo el retraso de este país con relación a países como Brasil, Uruguay, Chile, Colombia, Venezuela, México, etc.; podríamos enunciar una hegemonía simbólica en el pensamiento educativo, detentada por el imaginario del sistema educativo graduado obligatorio.

Para ir abriendo caminos en proyectos con los jóvenes consideramos imprescindible pensar en modificaciones estructurales que sostengan en oposición a la globalización neoliberal, un "altermundialismo" (Touraine, 2006; Fernández, 2007) con sentido regional, territorial y local, salvando también los escollos y desviaciones que pueden generarse en opciones no interpretadas con sentido crítico, que llevan a la segregación, los enfrentamientos y la violencia entre las culturas (Sen, 2007).

Sin una adecuada toma de posición frente a estas demandas plurales y complejas en un marco en donde el Estado se resiste y esquiva asumir nuevas responsabilidades sociales a largo plazo, es imperioso modificar no sólo los criterios fundantes que orientan la labor de las instituciones educativas sino fundamentalmente revitalizar las posibilidades de acompañar y potenciar los esfuerzos de los jóvenes en la lucha por superar la exclusión y la negación que la sociedad les hereda.

\section{Avizorando posibilidades de integración socio- educativa con y desde los jóvenes}

En un contexto de crisis, asistido por desigualdades incipientes y asediado por transformaciones económicas, productivas y labo- 
rales estrepitosas el problema constreñido a la superposición y la multiplicación de ofertas educativas destinadas a los jóvenes exigen una nueva toma de posición por parte de la administración pública y los dispositivos institucionales comprometidos con la gestión de las políticas sociales.

En el caso específico de los jóvenes la expansión de la pobreza, la exclusión social y el desgranamiento paulatino de las redes de contención institucional, civil y política recalan en la escasez de posibilidades de participación que ni el Estado ni el Mercado han podido procurar en virtud de la integración de las nuevas generaciones en la sociedad argentina.

Por un amplio periodo de tiempo, ha predominado en el campo de las políticas educativas la asunción pasiva y la expectación neutral ante la consagración de estas nuevas tendencias segregatorias y excluyentes, que inexorablemente han coadyuvado a ahondar la invibisilización de las verdaderas demandas y las realidades de los jóvenes.

Por este motivo, se debería tender hacia una propuesta educativa comprometida con la pluralidad de los contextos, capaz de involucrar a los actores juveniles y la comunidad educativa en el diseño, la viabilización y la evaluación de las políticas educativas y sociales, para así poder recuperar el contenido instituyente de las intervenciones pedagógicas e impulsar la construcción de estrategias y programas de formación adecuados (Sirvent y Llosa, 2001; Aparicio, 2006).

A nuestro juicio existen algunas ideas fuerzas que podrían orientar las transformaciones requeridas en el campo de la formación de jóvenes, a saber:

Considerar a los jóvenes no sólo como destinatarios de servicios sino fundamentalmente como actores estratégicos del desarrollo y referentes protagónicos de las acciones y las propuestas; para ello es imprescindible dotarlos de herramientas cognitivas, 
Construyendo puentes de integración entre la educación. Las perspectivas de empleo y los procesos de participación en Argentina - Pablo Christian Aparicio y Sergio Ignacio Carbajal

civiles y participativas sin las cuales es imposible implicarse objetivamente en la "toma de decisión";

Fortalecer la organización de las prioridades, los intereses y los anhelos colectivos a través del consenso que permita además la aproximación entre las diversas orientaciones, las líneas de acción y los referentes políticos ubicados en esta área de formación específica;

Desarrollar estrategias innovadoras en el ámbito de la formación no formal partiendo de la construcción y afianzamiento de redes institucionales y sociales basados en una concertación multisectorial;

Actualizar los mecanismos públicos de intervención y promoción social de la juventud partiendo de la asunción de la heterogeneidad inherente a los jóvenes. Para la consecución de esta finalidad se necesita apostar simultáneamente por el desarrollo teórico, metodológico y práctico de acciones pedagógicas atinadas y significativas que potencien la singularidad cognitiva y biográfica de los actores juveniles;

Ampliar los sistemas, las prácticas y los valores democráticos correspondientes a la ciudadanía y a la participación social en vistas a ampliar los procesos de integración y transformación social; 12

Consolidar los espacios colectivos de aprendizaje favoreciendo la intervención y el aporte de agentes externos provenientes de la sociedad civil, como por ejemplo los sindicatos, las ligas de familias, los centros vecinales, los clubes deportivos, las iglesias, los centros de desempleados, los hospitales, etc.;

12 Por su parte Touraine (1999), en una toma de posición crítica frente a las propuestas políticas públicas prevalecientes en el ámbito de juventud, comenta que, "en vez de buscar la integración social de los jóvenes pensando en la paz social, más que en los propios jóvenes, hay que fortalecer en éstos la capacidad de ser actores de su propia vida, capaces de tener proyectos, de elegir, de juzgar de modo positivo o negativo, y capaces también, más sencillamente, de tener relaciones sociales, ya se trate de relaciones de cooperación, de consenso o conflictivas." Op. cit. pág.77. 
Considerar los aspectos vinculados al género, la idiosincrasia étnica-cultural, el nivel socioeconómico, el lugar de residencia y el capital cultural acumulado y detentado por los padres como indicadores preponderantes de la condición de vida de las personas y, por ende, también elementos sustanciales para la elaboración eficiente de estrategias de formación y de apoyo socioeducativo; y

Generar una propuesta educativa fundada en los jóvenes, dotada de las condiciones y potencialidades necesarias para amalgamar sinérgicamente el aporte y el trabajo desarrollado por las demás carteras políticas públicas y ministeriales, las instituciones y los sectores -tanto civiles como privados- que actúan en el ámbito de la formación y atención de los jóvenes.

\section{A modo de conclusión}

El modo y velocidad en que se transformó el Estado y la sociedad en su conjunto a partir de los cambios estructurales efectuados entre los 80-90 en Argentina pueden dar cuenta de la nueva y problemática situación histórica que se abre ante los jóvenes.

Y es desde este prisma, desde donde indefectiblemente deben leerse los cambios políticos, sociales y educativos acometidos, así como también las consecuencias que estos trajeron aparejadas para las nuevas generaciones en términos de una mayor exclusión, indefensión y carencia de medios de referencia.

El ápice estructural de la desigualdad socioeconómica, la expansión de la pobreza, el desempleo abierto, la informalidad laboral y el desdibujamiento de referentes e interlocutores políticos e institucionales válidos puso en cuestión la legitimidad del nuevo modelo de desarrollo y concretamente interpeló el aporte de la educación como medio genuino de integración social y potenciación de las competencias, disposiciones y herramientas sociales y cognitivas para afrontar los desafíos de la inclusión en todos los campos de la vida social. 
En este escenario, la visibilización socioeducativa de los intereses y las demandas de los actores juveniles tan determinante y carente tendría que basarse en una asunción crítica de las condiciones de vida actuales y complejas que facilite el desarrollo idóneo de propuestas de inclusión social y de empoderamiento de los grupos más desmedrados.

La convergencia de intereses, la concertación de prioridades políticas y la armonización de fuerzas sociales transformadoras requieren indudablemente la disposición de medios, tiempos y estructuras de trabajo compartidas que favorezcan la contextualización de las propuestas educativas en consonancia a las necesidades, las limitaciones y las fortalezas de los diferentes escenarios formativos.

La validación y canalización pedagógica adecuada de las experiencias que los jóvenes realizan en el ámbito social, cultural, político y laboral reclaman una mejor articulación entre la teoría y la práctica educativa. Vale decir, el conjunto de estas experiencias y aprendizajes que se realizan al margen de los centros de formación requieren un mejor andamiaje tendiente a reconciliar: a) el saber oficial impartido desde las escuelas, b) el bagaje cognitivo, experiencial y social acumulado, c) las condiciones estructurales del contexto histórico social de vida, e) las demandas, las posibilidades y las perspectivas del entorno familiar y colectivo, y f) las disposiciones subjetivas, los proyectos biográficos y los itinerarios educativos.

En la síntesis de las demandas sociales y el aproximamiento vinculante entre los actores e instituciones involucradas en el ámbito de la formación se podrá avanzar en la construcción de un proyecto pedagógico más democrático y sustentable con posibilidades de contrarrestar la exclusión, los riesgos y la vulnerabilidad socioeconómica que hoy interpelan la cotidianidad de los grupos juveniles y resignifican el propósito fundante de los procesos educativos. 


\section{Bibliografía}

Álvarez, C. y Aparicio, P. (2000): Die Öffnung der Schule und der Hoffnung der Schule: Jugendhilfe in den lateinamerikanischen Schulen. (Chile und Argentinien), Biblioteca de la Universidad de Tübingen. Consultado el 4 de diciembre de 2008. [En red]. Disponible en:http://w210.ub.unituebingen.de/dbt/volltexte/2003/873/pdf/Oeffnung_der_Schule.pdf

Aparicio, P. Ch. (2003): Jóvenes, trabajo y educación. Intrincados dilemas de una tríada estratégica. En D. Michelini (Edit.), Riqueza-Pobreza: una perspectiva latinoamericana (pp. 45-71). Río Cuarto: Ediciones ICALA.

(2005): Los jóvenes, educación y política de juventud en Argentina. Restricciones y desafíos de la integración social en el contexto de la globalización [Versión electrónica]. Educere - Revista Venezolana de Educación N 30 Año 9 (pp. 287-296) http://www.saber.ula.ve/db/ssaber/Edocs/pubelectronicas/educere/vol9num30/articulo1.pdf

(2005b): Los jóvenes frente al impacto de la política educativa argentina. El antes y después de una reforma ambiciosa e inconclusa). Revista Umbral 2000 n 17 [Revista virtual], Chile http://www.reduc.cl/reduc/estudio17.htm

(2006): El trabajo infantil y el trabajo juvenil entre la exclusión y la pobreza. Un aporte socio-educativo. En P. Aparicio (Edit.), Niños y jóvenes en la encrucijada de la exclusión (pp.69-86). Río Cuarto: Fundación ICALA e.V.

(2007): Gioventù e giovani in America latina: Le sfide dell囚educazione di fronte all囚impronta della pluralizzazione e della segmentazione sociale, Rivista Internazionale di Edaforum: Focus on Lifelong Lifewide Learning Anno 2 Numero 8 http://rivista.edaforum.it/numero8/monografico_gioventu. html

(2007b): La Política Educativa Argentina y el incremento de la desocupación juvenil. Orientaciones y estrategias alternativas de integración socio-educativa desde el aporte de las Políticas de Transición y la Pedagogía Social Alemana. Tübingen: Archivos de la Biblioteca de la Universidad de Tübingen.

(2007c): “Los jóvenes y la vertiginosa transformación educativa, social y laboral. Una aproximación socio educativa a la realidad de la/os jóvenes argentina/ os", en Studi sulla Formazione, Anno IX 2006, Univeristá di Firenze, pp. 86-106. 
Construyendo puentes de integración entre la educación. Las perspectivas de empleo y los procesos de participación en Argentina - Pablo Christian Aparicio y Sergio Ignacio Carbajal

(2008): Jóvenes, educación y sociedad en América Latina: Los retos de la integración en un contexto de creciente pluralización cultural y segmentación socioeconómica. En P. Aparicio \& D. de la Fontaine (Coord.). Diversidad cultural y desigualdad social. Desafíos de la integración global. El Salvador, Heinrich Böll Stiftung, 155-198.

Bauman, Z. (1999): La Globalización. Consecuencias Humanas. Buenos Aires: Fondo de Cultura Económica.

(2003). Modernidad líquida. Buenos Aires: Fondo de Cultura Económica.

Beck, U. (1990): Risikogesellschaft. Auf dem Weg in eine andere Moderne. Frankfurt am Main: Suhrkamp.

Bourdieu, P. (1998): Gegen Feuer, Konstanz: UVK Verlagsgesellschaft $\mathrm{mbH}$.

(1998b): “La esencia del neoliberalismo", en Le Monde Diplomatique, $\mathrm{N}^{\circ} 528$, París.

(1999): Meditaciones pascalianas. Barcelona: Anagrama.

Carbajal, S. (2005): Protagonismo en contexto de exclusión. En Zaffaroni y equipo de cátedra (Comps.) Jóvenes protagonistas Salta 2004 (pp. 35-39) Salta: Editorial Milor.

CEPAL-CELADE (2000): Juventud, población y desarrollo en América Latina y el Caribe. Santiago de Chile: CEPAL.

CEPAL, AECID \& OIJ (2008): Juventud y cohesión social en Iberoamérica. Un modelo para armar. Santiago de Chile, CEPAL.

Fernández, A.M. (2007): Las lógicas colectivas. Buenos Aires: Editorial Biblos.

Filmus, D. et al. (2001): Cada vez más, necesaria, cada vez más insuficiente. Escuela media y mercado de trabajo en épocas de globalización, Buenos Aires.

Filmus, D. y Miranda, A. (1999): "América Latina y Argentina en los 90: más educación, menos trabajo=más desigualdad", en D. Filmus (comp.), Los noventa. Política, sociedad y cultura en América Latina y Argentina de fin de siglo. Flacso-Eudeba Editores, Buenos Aires.

Frigerio, G. (2002): “Entre la repetición y la ocasión de lo nuevo. El derecho a inventar otra cosa" en UNESCO: Qué es la educación secundaria para el siglo XXI, Oficina Regional de Educación 
de la UNESCO para América Latina y el Caribe (UNESCO), Santiago de Chile.

Gallart, M. A. (2001): La formación para el trabajo y los jóvenes en América Latina CEPAL-GTZ, Chile.

Jacinto, C. (2003): “Nueva institucionalidad en la formación para el trabajo de jóvenes en América Latina: alcances y límites de las nuevas estrategias". Congreso Latinoamericano de Trabajo, La Habana, Cuba.

Lechner, N. (1999): Las condiciones de la gobernabilidad democrática en América Latina de fin de siglo. En Filmus, D. (comp.), Los noventa. Política, sociedad y cultura en América Latina y Argentina de fin de siglo. Buenos Aires, Flacso/Eudeba, pp. 11-23.

Mancebo, M. (1999): "La sociedad Argentina de los 90: Crisis de socialización" en Filmus (Comp.): Los noventa. Política, sociedad y cultura en América Latina y Argentina de fin de siglo, pp. 177199.

Minujín, A. (1999): ¿La gran exclusión? Vulnerabilidad y exclusión en América Latina. Revista Iberoamericana de Educación, OEI, Madrid.

Naciones Unidas (2005): Objetivos de desarrollo del milenio. Una mirada desde América Latina y el Caribe. Santiago de Chile: Naciones Unidas.

OIJ (Organización Iberoamericana de la Juventud) (2004): Las encuestas de jóvenes en Iberoamérica. Un recuento de experiencias recientes. Análisis de las encuestas nacionales de jóvenes de Guatemala, Colombia, Chile, España, México y Portugal (1997-2000). Madrid: OIJ.

(2004): Panorama laboral 2004. América Latina y el Caribe. Lima: Oficina Regional para América Latina y el Caribe.

(2005): El empleo de los jóvenes: vías para acceder a un trabajo decente. Ginebra: Organización Internacional del Trabajo.

(2004b): Regional Report on the Achievement of the Millennium Development Goals in the Caribbean Community. Mona: Universidad de las Indias Occidentales.

PNUD (Programa de las Naciones Unidas para el Desarrollo) (2004). Informe sobre desarrollo humano 2004. Madrid: Ediciones MundiPrensa Libros S.A. 
Construyendo puentes de integración entre la educación. Las perspectivas de empleo y los procesos de participación en Argentina - Pablo Christian Aparicio y Sergio Ignacio Carbajal

Oszlak, O. (2003): El mito del Estado mínimo: una década de reforma estatal en la Argentina. Desarrollo Económico. Revista de Ciencias Sociales, vol. 42, No 168, Buenos Aires, IDES, pp. 519-543.

PROSUR 2005 (2005): Políticas locales de Juventud. Experiencias en el Cono Sur. Buenos Aires: Friedrich Ebert Stiftung.

Puiggrós, A. (Comp.) (1999): En los límites de la educación, Buenos Aires 1999.

Puiggrós, A. y Dussel, I. (1999): "Fronteras educativas en el fin de siglo: Utopías y distopías en el imaginario pedagógico" en Puiggrós (Comp.) Op. cit. pp. 7- 50.

Riquelme, G. (2004): La educación secundaria antes y después de la reforma. Buenos Aires: Miño y Dávila Editores.

Rodríguez, E. (2002): Documento de base. Insumos iniciales para el diseño del libro blanco sobre políticas de juventud en Iberoamérica. Texto preparado para la Secretaría General de la Organización Iberoamericana de Juventud (OIJ) como Documento de Base; Uruguay.

Rodríguez, E. (2005): Organizaciones y movimientos juveniles en América del Sur: estado de situación y bases para un programa de fortalecimiento institucional. Montevideo: Celaju /Unesco/Banco Mundial.

Salvia, A. (2002): La estructura social del trabajo en Argentina: desempleo, subempleo y precariedad laboral. Documento de Investigación AE/Notas/SL01. Universidad Católica Argentina: Área Económica, Departamento de Investigación Institucional.

Salvia, A. y Tuñón, I. (2002): Los jóvenes trabajadores frente a la Educación, el Desempleo y el deterioro social en la Argentina. En Friedrich Ebert Stiftung (Edit.): Jóvenes trabajadores en el Mercosur y Chile: Causas, Consecuencias y Políticas (pp. Chile: Friedrich Ebert Stiftung).

Sen, A. (2007): Identidad y violencia. Buenos Aires: Katz Editores.

Sirvent, M. y Llosa, S. (2001): Jóvenes y adultos y educación en la Ciudad de Buenos Aires. Revista de Investigaciones del Instituto de Ciencias de la Educación, Buenos Aires.

Sottoli, S. (1999): Sozialpolitik und entwicklungspolitischer Wandel in Lateinamerika. Konzepte und Reformen in Vergleich. Opladen: Leske + Budrich. 
Tedesco, J. C. (2004): “Desafíos de la educación secundaria en América Latina", en C. Jacinto (coord.), ¿Educar para qué trabajo? Discutiendo rumbos en América Latina. Editado por La Crujía, Ministerio de Educación, Ciencia y Tecnología; Ministerio de Trabajo Empleo y Seguridad Social y RedEtis, Buenos Aires.

Tenti Fanfani, E. (2003): Educación media para todos. Los desafíos de la democratización del acceso. Buenos Aires: IIPE/UNESCO, Fundación OSDE: Altamira.

Tiramonti, G. y Minteguiaga, A. (2004): Una nueva cartografía de sentidos para la escuela. En G. Tiramonti (Comp.): La trama de la desigualdad educativa (pp. 101-117). Buenos Aires: Manantial.

Touraine, A. (1999): ¿Podremos vivir juntos? Brasil: Fondo de Cultura Económica.

(2006): Un nuevo paradigma para comprender el mundo de hoy. Paidós. Argentina.

UNESCO (2004): La conclusión universal de la educación primaria en América Latina: iestamos realmente tan cerca? Informe Regional sobre los objetivos de desarrollo del Milenio vinculados a la educación. Santiago de Chile: Oficina Regional de Educación de la UNESCO para América Latina y el Caribe (OREALC).

(2006): EFA Global Monitoring Report. Strong foundations. Early childhold care and educacion: http://unesdoc.unesco.org/ images/0014/001477/147794E.pdf Rev. 24.08.2007

Zaffaroni, A. (2006): Representaciones y propuestas sobre educación de jóvenes salteños. En A. Zaffaroni y equipo de cátedra (Comps.), Ámbitos del protagonismo juvenil (pp. 207-217). Salta: Editorial Milor.

Zaffaroni, A. y Carbajal, S. (2008): Jóvenes en contextos de precariedad socioeconómica y de desigualdad educativa en el Noroeste Argentino. En P. Aparicio \& D. de la Fontaine (Coord.). Diversidad cultural y desigualdad social. Desafíos de la integración global. El Salvador, Heinrich Böll Stiftung, pp. 199-226. 